\title{
Delivering Gigabit Capacities to Passenger Trains Tales from an Operator on the Road to $5 \mathrm{G}$
}

\author{
Nima Jamaly, Stefan Mauron, Ruben Merz, Adrian Schumacher, Daniel Wenger ${ }^{\dagger}$ \\ Swisscom Ltd., Switzerland \\ \{nima.jamaly, stefan.mauron, ruben.merz, adrian.schumacher, daniel.wenger1\}@swisscom.com
}

\begin{abstract}
Delivering reliable and high-capacity Internet connectivity to high-speed train users is a challenge. Modern railway cars act as Faraday cages and a typical train consist comprises several hundreds of users moving at high velocity. Furthermore, with the global availability of fourth generation (4G) Long Term Evolution (LTE), user expectations have dramatically increased: it is expected to be online anytime and anywhere. Demand for mobile highcapacity is being driven by video and music streaming services, for lower latency and higher availability by gaming, and for more reliability and even uplink capacity by mission critical applications. Finally, the life-cycle of the railway industry is much longer than for telecommunications, which makes supporting $5 \mathrm{G}$ challenging. In this paper, we survey the challenges associated with delivering high-capacity connectivity to train users, describe potential options, and highlight how a leading western European operator is tackling these challenges and preparing for $5 \mathrm{G}$ and beyond.
\end{abstract}

\section{INTRODUCTION}

Delivering reliable mobile connectivity to train users has always been challenging. First, railway cars with their metallic bodies and low emissivity (LowE) coated glass windows for thermal isolation act as Faraday cages and cause high signal attenuation that need compensation. For the typical frequency range of $800 \mathrm{MHz}$ to $2600 \mathrm{MHz}$, measurements have shown an attenuation of 25 to $30 \mathrm{~dB}$ [1]. Second, a train can transport several hundreds to more than one thousand users. In a typical western European country, this represents hundreds of Long Term Evolution (LTE) active user-equipments (UE), traveling at speeds up to $330 \mathrm{~km} / \mathrm{h}$. Advanced Doppler compensation algorithms are required, and network dimensioning and optimization is non-trivial because the high rate of handover and cell-reselection caused by high train velocities need to be managed carefully: for example,

\footnotetext{
${ }^{\dagger}$ Authors in alphabetical order.
}

cell-reselections or handovers can take place every 40 seconds for up to 1200 users simultaneously [2].

Finally, the deployment of $4 \mathrm{G}$ has fueled an increasing demand for high-capacity mobile connectivity. Mobile users expect unlimited access to video and music streaming services, instant access to social media services, and reliable connectivity for gaming services. These expectations remain when users are commuting or traveling. For instance, in Switzerland, with the densest railway network in Europe, about $720 \mathrm{~km}$ of railway tracks are used daily by more than one million commuters. This puts a significant pressure on network and train operators to provide adequate connectivity [3]. Train and network operators around the world are trialing next generation solutions: for example in France [4], in China [3], but also in Germany, UK, or Japan.

Before describing our contribution, two more aspects need to be discussed. The first is associated with the major strategy used by service providers and railway operators to provide connectivity to train users by deploying on-board equipment, for instance, signal repeaters, or $\mathrm{Wi}-\mathrm{Fi}$ routers backhauled via the cellular network. This strategy is threatened in two ways: (1) The use of multiple-input multiple output (MIMO) systems popularized in $4 \mathrm{G}$ and improved in fifth generation systems (5G) with higher order MIMO, the use of both time-division duplexing (TDD) and frequency-division duplexing (FDD) in $5 \mathrm{G}$, and the use of millimeter-wave spectrum in $5 \mathrm{G}$, dramatically increasing system complexity and cost for on-board equipment; (2) upgrading this equipment to keep pace with standardization progress is impeded by the life-cycle duration difference between the railway industry and the telecommunications industry: typically, train infrastructure has life-cycles of tens of years, whereas wireless communications infrastructure is upgraded every few years (mobile network operators started 
LTE services late 2012 and already upgraded to LTE Advanced and LTE Advanced Pro). To upgrade railway infrastructure with the newest wireless communications infrastructure every three to five years is for train operators a costly if not impossible mission. Another aspect is tied to $5 \mathrm{G}$, with which service providers will not only serve broadband applications, but also - enabled by network slicing - future railway mobile communication services (FRMCS) [5].

Hence, in this paper, our contribution is threefold. First, a survey of the challenges associated with supporting high-capacity train connectivity is presented in Section III. Then, a state of the art overview of how these challenges are addressed by a majority of operators is given in Section III. However, this current approach is threatened by the arrival of 5G. Finally, our third contribution in Section IV] is a first look at how a leading western European operator is revisiting these approaches to tackle the foregoing challenges, and preparing for $5 \mathrm{G}$. Section $\mathrm{V}$ concludes the paper.

\section{What ARE The Challenges of Modern RAILWAY CONNECTIVITY?}

In this Section, we describe the most relevant challenges faced by service providers and train operators to provide high-capacity connectivity to train users.

\section{A. Railway Car Penetration Loss}

Modern railway car hulls are typically built of metal, creating a Faraday cage. While the window apertures potentially allow signals to get into the railway car, this effect is negated by the use of socalled Low-E coated glass windows [1].

\section{B. High-Velocity}

Intercity high-speed trains in Europe travel between 160 and $330 \mathrm{~km} / \mathrm{h}$. In Asia similar velocities are used for commercial operation. High velocity results in strong Doppler effects, creating various performance degradations [4], [6]: Firstly, it creates distortions on the signal at the receiver that need to be compensated. Secondly, the Doppler effect is directly related to the coherence time of the propagation channel. It is a measure of how stable the channel remains over time. Fast evolving channels are more difficult to estimate (e.g., averaging over time is limited) and result in lower data rates. Last, a small coherence time highly decreases the potential of feedback-based pre-coding schemes for MIMO systems. High velocity also increases the rate of handovers and cell-reselections [2]. If the network is not planned accordingly for large train consists (see below), this can result in performance anomalies because of control-plane overload, temporary stalling at the transport layer, and in the worst-case, increased control-channel loss.

\section{Large Number of Co-Located Users}

In a cellular network, users share the available sector bandwidth and control-plane capacity. Trains cluster a large number of active terminals traveling simultaneously through the network. For instance, a typical 400 meters long inter-city train consist can carry about 1200 passengers during rush hours. With trains crossing or at railway stations, even a larger number of active users per sector may have to be considered. Such large numbers of users require operators to over-dimension their infrastructure in order to not decrease service quality and throughput.

\section{Supporting MIMO End-to-End}

MIMO is the key feature to support high-capacity connectivity via spatial multiplexing schemes, and improve the overall robustness of the connectivity via diversity [6]. Now, in case of a train scenario, with e.g., a signal repeater and radiating cables (or antennas) deployed onboard the railway car (see Fig. 2b), it is critical that the entire RF chain supports MIMO. If not, it will mostly prevent the use of spatial multiplexing or diversity. While $2 \times 2$ MIMO support has been validated [7], $4 \times 4$ MIMO or massive MIMO schemes will be challenging. The latter is particularly valid in case channel state information at the transmitter is required, or if more than two receive antennas are needed.

\section{E. Life-Cycle Industry Differences}

A major challenge for active solutions in railway cars is the difference between technology cycles of telecommunication infrastructure (3 to 5 years) and the life-cycle of railway infrastructure (15 to 20 years). It is not feasible and sometimes not possible to replace connectivity-related equipment in railway cars every couple of years. Indeed, railway cars traditionally require an in-depth revision every 15 to 20 years. Only during such events can extensive changes e.g., in cabling or communication equipment, be undertaken. 


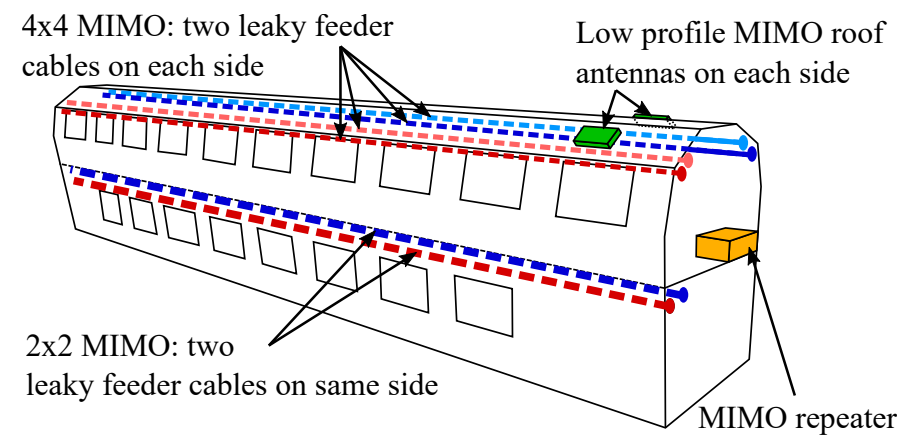

Fig. 1. Railway car equipped with roof antennas, a repeater, and leaky feeder cables. Not shown are interconnecting jumper cables.

\section{F. Limited Space and Flexibility}

Whichever active communications equipment is installed inside a railway car, it will require one or several external antennas. Ideally, they are installed on the railway car's roof or side of the roof. However, depending on the railway car's size (considering double-deck railway cars), the clearance gauge does not allow for large antennas.

Furthermore, limited space, energy and airconditioning along with an extended operation temperature range poses challenges for active communications equipment within railway cars. Cabling between different components such as e.g., antennas and communications equipment becomes very difficult (thermal insulation, railway car structure, interior design), and is very limited between railway cars.

\section{How ARE CHAllenges AdDRESSED?}

In this Section, we describe how challenges listed in Section II are typically addressed by service providers and train operators.

The most important challenge to address is to compensate the railway car penetration loss, followed by - in the context of $4 \mathrm{G}$ and $5 \mathrm{G}$ network deployment - the need to support end-to-end MIMO. The most popular solution pursued to combat the penetration loss is by outfitting the railway car with active equipment such as repeaters or relays [8]. This implies the installation of outside roof antennas for the backhaul (or donor link) and antennas or radiating cables (also known as leaky feeder cables, see [7]) inside the railway car for signal distribution (see Fig. 1). We describe these options in more details in the following Section.

\section{A. Repeater, Relay Node and Moving Cell}

A repeater amplifies the input port signal and sends it out on the output port. While operating at the physical layer, modern repeaters are implemented in the digital domain. Their main advantage is the independence of the radio access technology (RAT) and therefore transparency to wireless communications equipment. However, in order to prevent feedback loops and the resulting signal degradation or amplifier saturation, a repeater requires a high RF isolation between the inside radiating element(s) to the outside antenna(s) (the amplified signal is on the same frequency as the donor). The current observed $\mathrm{RF}$ isolation of repeater equipped railway cars is between 60 to $80 \mathrm{~dB}$. Another well-known disadvantage is that repeater deployments can be responsible for large noise-floor elevation, which can severely limit uplink capacity [9, Section 2.1]. Furthermore, due to signal filtering in the repeater, a signal delay (filter group delay) is introduced into the system. Some repeaters allow to keep this delay below the length of the cyclic prefix of LTE to prevent interference between the inside and outside signals. If the RF isolation is not sufficiently high, this signal delay together with the ratio of repeater gain to railway car RF isolation causes unwanted self-interference.

A relay-node $(\mathrm{RN})$ is another option, similar to repeaters, but operating on the upper layers. An RN can solve some of the problems observed with repeaters. Due to signal decoding and re-encoding, the re-transmitted signal is clean of noise and possible interference. Also, numerous users can be aggregated to significantly save on control-plane signaling, especially during handovers. However, RNs also require a high system isolation in case the same bands are used. Further disadvantages are the dependency on RAT releases and feature support, resulting in RN firmware or hardware upgrades, and the use of more space and electrical power. From a $3 \mathrm{GPP}$ point of view, RNs are only standardized for LTE and without mobility support. To the best of our knowledge, today no LTE RN is in operation in the railway context.

A further evolution of the RN concept is the socalled moving-cell, where UEs on the mobile link do not experience handovers or cell-reselection. Only the donor link handles these events. The movingcell can help solving the challenge related to a large number of users. However, this implies that a large part of the protocol stack of the underlying airinterface(s) be implemented in the active equipment. Because typical deployments today need to support multiple RAT generations, from $2 \mathrm{G}$ to $5 \mathrm{G}$, we are not aware of any moving cell in operation today. 
Common for repeaters and RNs is the problem with concurrent operation of TDD and FDD links on the same RF transmission systems, which dramatically increases the complexity of isolation and filtering.

\section{B. On-Board Wi-Fi}

Another way of providing wireless connectivity inside railway cars is by means of an on-board Wi$\mathrm{Fi}$ access point. The backhaul link of course uses cellular connectivity, but the mobile link is using WiFi. Contrary to repeater or RN equipment, such a system is much more independent of the railway car hull isolation, because different frequency bands are used. Although care needs to be taken between the ISM band at $2.4 \mathrm{GHz}$ and LTE bands 7 or 38 around 2.6 GHz. Regarding the deployment of $\mathrm{Wi}-\mathrm{Fi}$ inside railway cars, it should be noted that a performanceoriented system must support the UNII $5 \mathrm{GHz}$ band and recent standards (IEEE 802.11ac or 802.11ax).

Deployments of mobile routers with $\mathrm{Wi}-\mathrm{Fi}$ in the railway cars may prove more advantageous from a cost, power consumption, and evolution point of view. However, they do not solve the need for a highquality backhaul, antennas on the roof, or radiating cables or antennas inside the railway car. While it is possible to use Wi-Fi calling, it may not work for all passengers, and an end-to-end quality of service control is also not straightforward to achieve. Additionally, because many users make heavy use of Wi-Fi tethering, the 2.4 and $5 \mathrm{GHz}$ bands are already fairly crowded.

\section{Donor Link: MIMO Roof Antennas}

All active communications equipments discussed so-far require donor link antennas, ideally installed on the railway car roof. As discussed in the previous Section II-F, the clearance gauge may not allow for large antennas on the roof. For example, to solve this challenge for train operators, we developed a lowprofile MIMO antenna together with Huber+Suhner for double-deck railway cars where a maximum height of $40 \mathrm{~mm}$ is possible, see Fig. $2 \mathrm{a}$.

At least two antenna elements are required to support $2 \times 2$ MIMO. For higher order MIMO or diversity, more antenna elements need to be installed. Furthermore, there are very often constraints regarding the installation locations on the roof, available installation space, and orientation. Also, care must

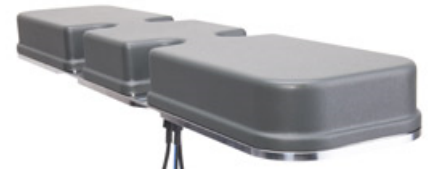

(a)

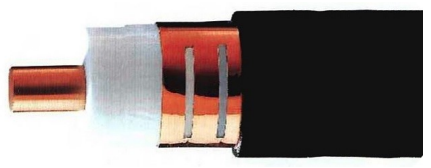

(b)
Fig. 2. (a) Low-profile $2 \times 2 \mathrm{MIMO}$ roof antenna for $790-2700 \mathrm{MHz}$ and 4.9-6.4 GHz. (b) Example of a radiating cable.

be taken to ensure that the rooftop antenna elements' position allows for a high-rank propagation channel.

\section{Propagation Inside Railway Cars: MIMO Radi- ating Cables}

Inside railway cars, radiating elements also need to be deployed. Radiating cables (see Fig. 2b) are typically used: they are easy to install, along the length of the railway car interior, either under the ceiling, along the side, or under the floor; radiating cables also create a more uniform signal along the railway car compared to antenna deployments; finally, radiating cables can be easily hidden from passenger view. Indeed, antenna deployments have sometimes created controversies with passengers uncomfortable with radio-wave deployments.

State of the art radiating cables support frequencies in the range of a few hundred $\mathrm{MHz}$ up to $2600 \mathrm{MHz}$. However, more wideband support on a single cable appears quite challenging. Support of both, cellular frequencies and the ISM band at $5 \mathrm{GHz}$, would require the deployment of two-cables. Deployment of MIMO spatial multiplexing requires additional cables as well, which has been validated with extensive measurements. In [7] we demonstrated that $2 \times 2$ MIMO as well as $4 \times 4$ MIMO can be supported.

\section{E. What are Most Operators Using Today?}

The majority of operational deployments today are building on repeaters or on-board Wi-Fi, or a combination of both. Antennas or radiating cables are used within railway cars. And while solutions are available for supporting $2 \times 2 \mathrm{MIMO}$ end-to-end, they are not widely deployed because of life-cycle challenges.

Indeed, the life-cycle challenge is not solved at all by current solutions, and its importance will further grow at the advent of $5 \mathrm{G}$ and the evolution of Global System for Mobile Communications Railway (GSM-R) [10] to FRMCS [5]. Hence, before moving on to the next Section where we briefly 
describe these challenges, we will in short outline some of the relevant specifics of 5G and FRMCS.

\section{F. The Rise of $5 G$}

New Radio (NR) is the successor RAT of LTE currently being specified by 3 GPP in Release 15 and 16. The first commercial network deployments have recently been switched on and early devices are expected to appear during the first half of 2019. With ultra lean transmission, latency optimized frame structure, massive MIMO and interworking between low and very high frequency bands (from $600 \mathrm{MHz}$ up to $86 \mathrm{GHz}$ [11]) it will dramatically increase datarates available to train passengers and applications. The latency improvements, the support of higherreliability and mission-critical applications, and the availability of network slicing will also be beneficial for train applications, especially in the context of FRMCS applications.

Deploying NR in millimeter-wave (mmWave) frequencies (e.g., $f>24 \mathrm{GHz}$ ) mandates the use of antenna arrays with e.g., 64-elements or more, adaptive beamforming, and TDD. Concurrently, a network densification is required due to increased free-space path loss and decreased signal penetration through obstacles.

In Europe, a focus on NR deployments at 3.5$3.8 \mathrm{GHz}$, using carrier bandwidths of $100 \mathrm{MHz}$ or more, TDD, and antenna arrays, followed by mmWave deployments is expected.

\section{G. Future Railway Mobile Communication System}

Focusing on Europe, currently many operators rely on their own GSM-R networks. They are primarily used to transport voice communication (point-topoint, group, broadcast and emergency calls) and most importantly, data traffic of the European Train Control System (ETCS) [12]. Today, ETCS Level 2 is in use, but the change to level 3 is being evaluated. ETCS level 3 requires accurate and reliable positioning and relies on radio-based train spacing. This will support increasing the capacity of heavily loaded railway networks. Hence, many train operators plan a thorough digitization of end-to-end railway operations, with an outlook on autonomous train operation. Future railway operation demands for higher data rates and therefore new spectrum and a network densification leveraging synergies of the telecommunications industry. Thus, the railway industry, aligning with the telecommunications industry, is developing FRMCS in order to support this digitalization effort, and the eventual decommissioning of GSM-R. The first FRMCS trial implementations are expected to start around 2020. Therefore, in addition to provide mobile connectivity to train users, service providers will also likely have to consider the support of FRMCS services.

\section{BRINGING HIGH-CAPACITY AND}

\section{SUPPORTING 5G B Y DECOUPLING LIFE-CYCLES}

While on-board repeaters or Wi-Fi routers have become popular to deliver capacity for railway users, they have also become a bottleneck. Indeed, on-board equipment is responsible for the life-cycle coupling challenge. With the advent of $5 \mathrm{G}$, bringing spectrum at $3.5 \mathrm{GHz}$, a TDD air-interface and massive MIMO, a major equipment upgrade is necessary. Furthermore, 5G with its dual use of TDD and FDD, dramatically increases the requirements on on-board equipment.

Therefore, in order to reduce the life-cycle coupling challenge as much as possible, and to ensure that railway users can readily benefit from cellular infrastructure innovation, we have decided to move away from using on-board equipment. Instead, we have decided to promote and use so-called RF transparent Low-E windows [1] (hereafter RF transparent windows).

\section{A. RF Transparent Windows make On-board Equip- ment Obsolete}

In modern railway cars, the window panes have a thin metallic coated layer for enhanced thermal (infrared) insulation. However, such a metallic coating increases the RF transmission loss through them significantly (e.g., $25-30 \mathrm{~dB}$ [1]). Recently, by adjusting the coating pattern on the window panes, engineers have successfully reduced the transmission loss for sub $3 \mathrm{GHz}$ bands to only a couple of $\mathrm{dBs}$. Simulations and measurements of railway cars equipped with RF transparent windows in real conditions demonstrated a reduction of around $12-14 \mathrm{~dB}$ at $1.8 \mathrm{GHz}$ band [1], [13]. Using frequency selective surface concepts in electromagnetics, scientists are expected to come up with an improved coating pattern which could likewise serve to reduce the transmission loss for frequency ranges up to $6 \mathrm{GHz}$. 
As such, classic $2 \times 2$ or higher order MIMO spatial multiplexing is supported.

RF transparent windows are the crucial parts for supporting high-capacity for railway applications. In addition, earlier studies, for example [2], have clearly shown that even at the high-velocity characteristics of railway scenarios, ensuring a high signal to interference and noise ratio (SINR) is the key factor to benefit from high spectral efficiency offered by spatial multiplexing schemes. As such, we are also promoting the use of dedicated cellular infrastructure for railway coverage. This design choice is also guided by the upcoming requirements of FRMCS (see Section III-G).

\section{B. Dedicated Infrastructure for high SINR: the RF Corridor}

In order to increase SINR, a dedicated infrastructure is required, deployed as close as possible to the railway track, a so-called $\mathrm{RF}$ corridor. Indeed, tight emmission regulations prevent increasing existing sector power. A dedicated infrastructure is a form of sectorization and densification, which helps supporting large number of users. A dedicated infrastructure also enables specific optimization for railway scenarios, but also improved localization support (e.g., for FRMCS). Combined sectors can also be deployed to reduce cell-reselection and handover.

Distributed antenna systems (DAS) or cloud-RAN approaches can be used to build such infrastructure. DAS are favored in case of multi-operator support requirements. However, they often lack proper (digital) interworking with base-station equipment to support the use of active antenna mechanisms or sophisticated MIMO algorithms.

An RF corridor may need integration in the existing macro-network. Various options are available, from using dedicated bands, to benefiting from improved spatial reuse by beamforming (possible with NR) or heterogeneous network algorithms.

\section{Reflecting Panels}

Our early simulations of RF corridor with RF transparent window-equipped trains have highlighted one potential issue: in case antennas are installed near the railroad, wide angles of incident wave to the window panes become unavoidable, especially in scenarios with poor scattering and reflections provided by the surrounding environment. This configuration can

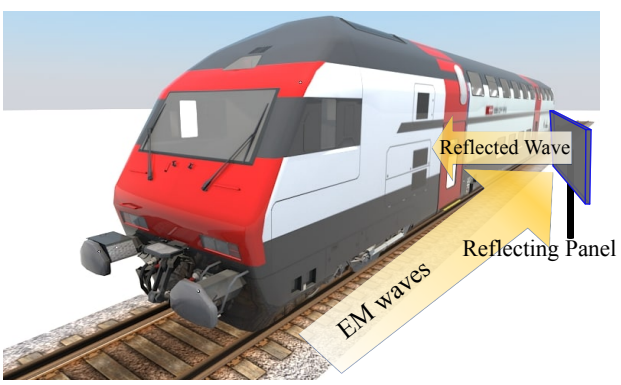

Fig. 3. Outlook of a reflecting panel in front of a railway car.

increase the penetration loss considerably (because of the significantly reduced cross-section area). To combat this performance anomaly, reflecting panels can be deployed along the railway tracks [14]: these conductive panels indirectly increase the cross section area of the window panes from the antenna standpoint. Fig. 3 illustrates such a scenario, where a simple flat conductive panel with $45^{\circ}$ angle towards the railway car is used to reflect RF energy towards the window panes. The exact gain of a reflecting panel on path loss strongly depends on its physical size and design.

In Fig. 4, we show the path loss between the ports of a base station antenna (with $20 \mathrm{dBi}$ gain), and an ideal dual-port omni-directional reference antenna (with $0 \mathrm{dBi}$ gain) on-board a typical railway car. The illustrated path losses at $2.6 \mathrm{GHz}$ are shown for vertical (VER) and horizontal (HOR) polarizations (with respect to the ground) and with and without a reflecting panel at a distance of $250 \mathrm{~m}$ from the antenna. The window panes used here are similar to those studied in [14] and scenarios without panel are referred to as line of sight (LOS).

Clearly, the impact of panels on the path loss is significant and rather independent of the traverse distance, as long as the traverse distance is a small fraction of the distance between the panel and the base station antenna.

\section{Outlook on Performance and Long-Term Spec- trum Evolution}

We have already validated the benefit of RF windows in a regular macro-network [1]. Throughout 2019, we will evaluate the improvements brought by deploying an RF corridor supported by reflecting panels. Early simulation results demonstrate that average spectral efficiencies can be doubled compared to regular macro-network coverage.

Looking forward, while many initial 5G deployments will begin with $3.5 \mathrm{GHz}$, systems in mmWave 


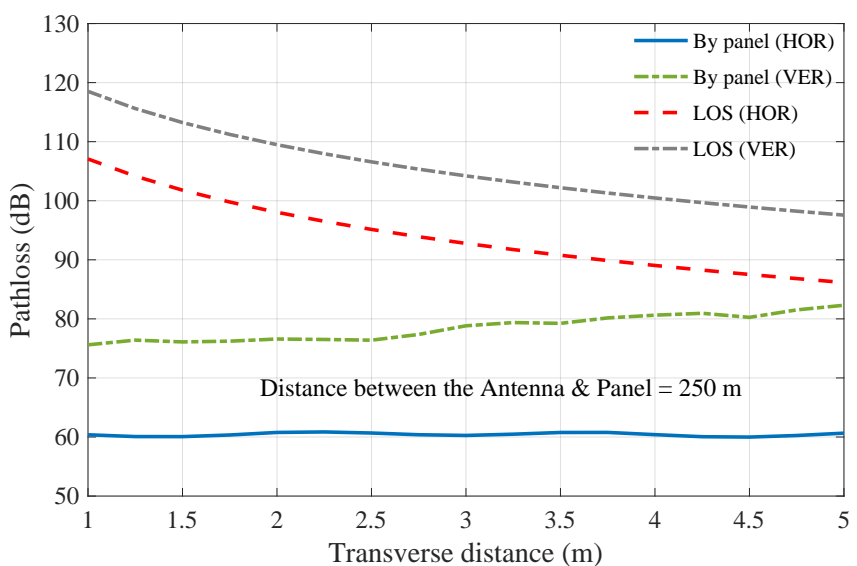

Fig. 4. Pathloss for the two polarizations with and without reflecting panel in an RF corridor scenario at $2.6 \mathrm{GHz}$. Gains of antennas are $\mathrm{G}_{\mathrm{tx}}=25 \mathrm{dBi} \& \mathrm{G}_{\mathrm{rx}}=0 \mathrm{dBi}$.

frequencies will eventually be deployed. To allow the use of such frequency ranges for train passengers, we expect to build on the existing RF transparent windows. Indeed, while RF transparent windows do not completely remove the life-cycle challenge, they reduce it significantly. Exchanging windows is much easier than a complete on-board RF installation. And recent demonstrations such as mmWave on-glass antennas are promising [15].

\section{CONCLUSION}

In this paper, we have reported on how a leading western European operator intends to evolve railway cars with RF transparent windows, and combine this approach with RF corridors and reflective panels to deliver very high capacity connectivity to train customers. This combination will allow for the support of $5 \mathrm{G}$ deployments up to $3.5 \mathrm{GHz}$ without any complex and costly on-board equipment. RF transparent windows have already been validated in the field. Their validation with an RF corridor and reflecting panels, in the context of early $5 \mathrm{G}$ deployments is ongoing. The use of mmWave for trains will also be addressed.

\section{REFERENCES}

[1] L. Burnier et al., "Energy saving glazing with a wide bandpass FSS allowing mobile communication: up-scaling and characterisation," IET Microwaves, Antennas \& Propagation, vol. 11, no. 10, pp. 1449-1455, Aug. 2017.

[2] R. Merz et al., "Performance of LTE in a High-velocity Environment: A Measurement Study," in Proceedings of the 4th Workshop on All Things Cellular. New York, NY, USA: ACM, 2014, pp. 47-52.

[3] B. Ai et al., "Challenges toward wireless communications for high-speed railway," IEEE Transactions on Intelligent Transportation Systems, vol. 15, no. 5, pp. 2143-2158, Oct 2014.
[4] F. Kaltenberger et al., "Broadband wireless channel measurements for high speed trains," in 2015 IEEE ICC, Jun. 2015, pp. 2620-2625.

[5] J. Moreno et al., "A survey on future railway radio communications services: challenges and opportunities," IEEE Communications Magazine, vol. 53, no. 10, pp. 62-68, Oct 2015.

[6] D. Tse and P. Viswanath, Fundamentals of Wireless Communication, May 2005.

[7] R. Merz et al., "A Measurement Study of MIMO Support with Radiating Cables in Passenger Rail Cars," in IEEE VTC Spring, Glasgow, Scotland, May 2015, pp. 1-5.

[8] D. T. Fokum and V. S. Frost, "A Survey on Methods for Broadband Internet Access on Trains," IEEE Communications Surveys Tutorials, vol. 12, no. 2, pp. 171-185, Second 2010.

[9] "An Assessment of the Effects of Repeaters on Mobile Networks," Ofcom UK, Tech. Rep. GTC-15-0007-D, Nov. 2015.

[10] “GSM-R - UIC - International union of railways.” [Online] Available: https://uic.org/gsm-r [Accessed: 2019-03-18]

[11] J. Lee et al., "Spectrum for 5G: Global Status, Challenges, and Enabling Technologies," IEEE Communications Magazine, vol. 56, no. 3, pp. 12-18, Mar. 2018.

[12] P. Winter, "The Project for a Unified European Train Control and Protection System (ETCS)," Rail International, no. 6-7, 1992.

[13] N. Jamaly et al., "Analysis and Measurement of Penetration Loss for Train Wagons with Coated vs Uncoated Windows," in EuCAP, Apr. 2018, p. 610 (5 pp.).

[14] N. Jamaly, S. Mauron, and A. Kishk, "Application of Reflecting Panels in Realisation of Antenna Corridor for Train Communications," in EuCAP, Apr. 2019, pp. 1-5.

[15] AGC, "Success with 5G Communications Using "Vehicle Glass Mounted Antenna" for 5G Connected Car," July 2018. [Online]. Available: http://www.agc.com/en/news/detail/ 1197413_2814.html [Accessed: 2018-12-14]

Nima Jamaly is a senior research engineer in Swisscom Innovations, Switzerland. During the last few years, he has also been a guest research scholar in Chalmers University of Technology, Sweden. His main areas of research are antennas and propagation.

Stefan Mauron holds an engineering degree in information-systems technology and in business administration, both from the University of Applied Sciences in Bern. Stefan is a principal product innovation lead for wireless access for the $5 \mathrm{G}$ program at Swisscom.

Ruben Merz is the lead system architect for the 5G program at Swisscom. Ruben earned his $\mathrm{PhD}$ and MSc from the School of Computer and Communication Systems at EPFL, and a master of advanced studies in management, technology \& economics at ETH Zürich

Adrian Schumacher [M'07] received his M.Sc. degree from the Royal Institute of Technology (KTH), Stockholm. He is a senior engineer at Swisscom Innovations. His research interests include digital signal processing, wireless communications, and mmWave.

Daniel Wenger received his B.Sc. degree in Electrical Engineering from University of Applied Science in Bern $(\mathrm{CH})$. He is a senior innovation engineer at Swisscom working in the $5 \mathrm{G}$ program. 